Conclusions Liver fibrosis, but not steatosis, was associated with more adverse cardiovascular health in young adults once known confounders such as adiposity were accounted for. Further follow up of this and similar cohorts will be important to determine whether cardiovascular health worsens over time in those with steatosis alone, once accounting for other cardiovascular risk factors.

Conflict of Interest None
193 EDOXABAN VERSUS WARFARIN ON STROKE RISK IN
PATIENTS WITH ATRIAL FIBRILLATION: A TERRITORY-
WIDE COHORT STUDY

${ }^{1}$ Che Him Dicken Kong, ${ }^{2}$ Jiandong Zhou, ${ }^{3}$ Sharen Lee, ${ }^{4}$ Keith Sai Kit Leung, ${ }^{5}$ Tong Liu, ${ }^{6}$ Abhishek C Sawant, ${ }^{7}$ John Corbelli, ${ }^{8}$ Abraham KC Wai, ${ }^{9}$ Carlin Chang, ${ }^{2}$ Qingpeng Zhang, ${ }^{5}$ Gary Tse. ${ }^{1} \mathrm{Li}$ Ka Shing Institute of Health Sciences and Chinese University Research Institute, Hong Kong, China; ${ }^{2}$ School of Data Science, City University of Hong Kong; ${ }^{3}$ Cardiovascular Analytics Group, Laboratory of Cardiovascular Physiology, Hong Kong; ${ }^{4}$ Aston Medical School; ${ }^{5}$ Tianjin Institute of Cardiology, Second Hospital of Tianjin Medical University; ${ }^{6}$ Banner University Medical Center, ${ }^{7}$ Buffalo VA Medical Center; ${ }^{8}$ The University of Hong Kong; ${ }^{9}$ Queen Mary Hospital

\subsection{6/heartjnl-2021-BCS.189}

Background In this territory-wide, observational, propensity score-matched cohort study, we evaluate the development of transient ischaemic attack and ischaemic stroke (TIA/Ischaemic stroke) in patients with AF treated with edoxaban or warfarin. Methods This was an observational, territory-wide cohort study of patients between January 1st, 2016 and December 31st, 2019, in Hong Kong. The inclusion were patients with i) atrial fibrillation, and ii) edoxaban or warfarin prescription. 1:2 propensity score matching was performed between edoxaban and warfarin users. Univariate Cox regression identifies significant risk predictors of the primary, secondary and safety outcomes. Hazard ratios (HRs) with corresponding 95\% confidence interval $[\mathrm{CI}]$ and $\mathrm{p}$ values were reported.

Results This cohort included 3464 patients $(54.18 \%$ males, median baseline age: 72 years old, IQR: 63-80, max: 100 years old), $664(19.17 \%)$ with edoxaban use and 2800 $(80.83 \%)$ with warfarin use. (Figure 1). After a median follow-up of 606 days (IQR: 306-1044, max: 1520 days), 91 (incidence rate: 2.62\%) developed TIA/ischaemic stroke: $1.51 \%(10 / 664)$ in the edoxaban group and $2.89 \%(81 / 2800)$ in the warfarin group. Edoxaban was associated with a lower risk of TIA or ischemic stroke when compared to warfarin (figure 2).

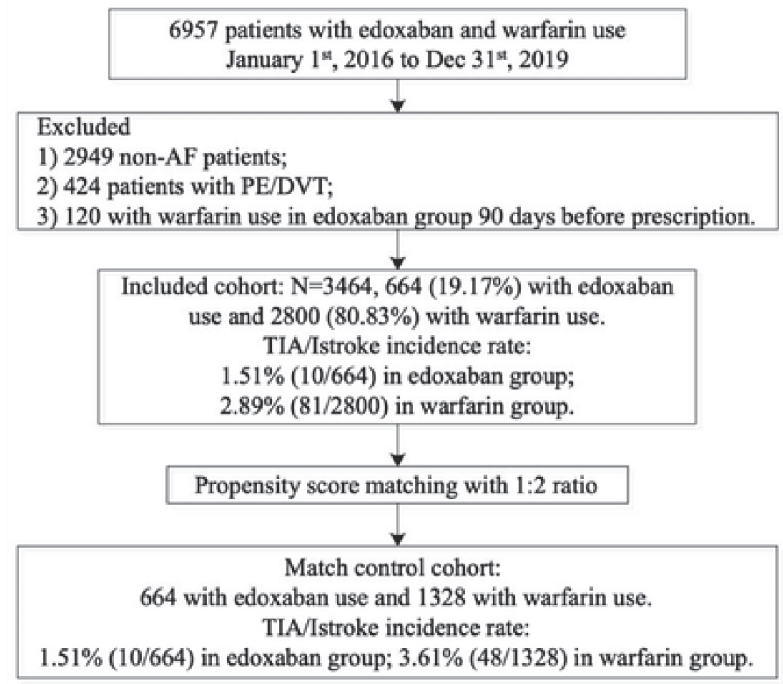

Abstract 193 Figure 1 

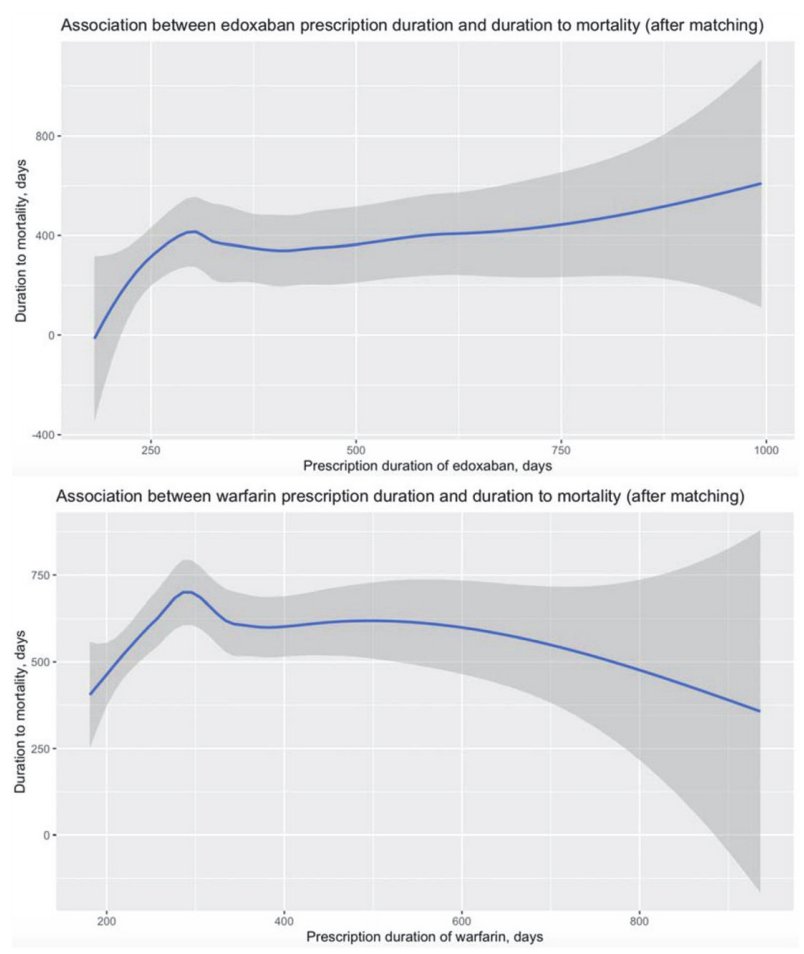

Abstract 193 Figure 2

Conclusions Edoxaban use was associated with a lower risk of TIA or ischemic stroke after propensity score matching for demographics, comorbidities and medication use.

Conflict of Interest None

\section{THE UCLPARTNERS PROACTIVE CARE FRAMEWORKS - OPTIMISING CVD PREVENTION POST COVID. INNOVATION TO RESTORE AND IMPROVE CARE IN THE HIGH-RISK CONDITIONS AND PREVENT HEART ATTACKS AND STROKES AT SCALE.}

${ }^{1}$ Matt Kearney, Deep Shah, Stephanie Peate, John Robson, Helen Williams, Sotiris Antoniou, Mandeep Butt, Aiysha Saleemi, Daphne Amevenu, Nausheen Hameed, Mohammed Khanji, Mairead McErlean, Laura Boyd. UCLPartners, London, UK

\subsection{6/heartjnl-2021-BCS.190}

Introduction COVID-19 has disrupted pathways of care for over 12 months. Primary care has transformed dramatically with much care being provided remotely. The COVID surges and vaccination programme have reduced capacity further. People with conditions such as hypertension, cardiovascular disease and diabetes depend on regular review and treatment optimisation to keep them well. There is a high risk that continued disruption to proactive care will drive an increase in exacerbations and complications. It is likely this will drive further waves of demand for urgent care over the coming months in primary care, in emergency departments and in hospital admissions.

Methods The team of GPs \& pharmacists at UCLPartners, with patient and public support, developed proactive care frameworks for six conditions including atrial fibrillation, hypertension, high cholesterol and type 2 diabetes mellitus. The frameworks focus on the 'how to' of delivering care in the new world of primary care post COVID-19. They are built on 4 principles: virtual where appropriate, use of the wider workforce, step change in self-management, and use of digital resources. For each condition, the frameworks include: risk stratification tools; pathways that support remote care and deploy staff such as healthcare assistants and social prescribers to systematically support education, self-management and lifestyle change; scripts, protocols and training to guide these staff in consultations; digital tools; and resources to support treatment optimisation. The frameworks include clinical and project management support for local pathway adaptation and implementation.

Results The frameworks have gained wide traction in primary care across England. There have been over 2,700 downloads of the search/stratification tools with evidence of implementation in several regions. In the UCLP geography, North East London and North Central London have adopted the frameworks for roll out across 475 GP practices and 2.8 million people. NHSEngland has now adopted the Frameworks as a key part of the NHS@Home programme with plans to support at scale national roll out. Evaluation is being commissioned.

Conclusions The UCLPartners Proactive Care Frameworks provide systematic, evidence-based support to restore services post COVID: stratifying so that higher risk patients can be prioritised and workload managed; maximising remote care; optimising personalisation and support for self-care. By using a population health management approach together with comprehensive resources to support clinical management in real world primary care, the frameworks provide a platform not just to restore services but to optimise treatment and outcomes in the high-risk conditions for CVD. The widespread national traction the frameworks are gaining suggests that despite the pandemic, this brings an opportunity to deliver the NHS Long Term Plan ambitions for CVD prevention and prevent 150,000 heart attacks, strokes and cases of dementia.

Conflict of Interest none

\section{NICE 95 AND NON ANGINAL CHEST PAIN: REASSURING PATIENTS WITHOUT INVESTIGATION}

Sarah Fellows, Agnieszka Kopanska, Badrinathan Chandrasekaran, Edward Barnes. Great Western Hospital NHS Foundation Trust, Swindon, UK

\subsection{6/heartinl-2021-BCS.191}

Background NICE 95 guidance (2016) tells us that patients presenting with stable non-anginal chest pain do not require further investigation where clinical suspicion of underlying coronary disease is deemed unlikely. This relies on the clinical acumen of the healthcare practitioner assessing patients with no pre-test probability score recommended in the current guidelines (a change from 2009 guidance). The European Society of Cardiology (ESC), however, recommend the use of a pre-test probability score to aid decision making in such cases. We believe that the current practice of reassuring this group of patients without investigation is safe and that a formalised pre-test probability score does not provide additional reassurance.Method:A retrospective single centre cohort study of all cases assessed in a face-to-face nurse led rapid access chest pain clinic (RACP) where patients were discharged without investigation. Pre-test probability scores were calculated according to the ESC chronic coronary syndromes guideline 